\title{
Avaliação da Necessidade da Solicitação de Exames Complementares para Pacientes Internados em Unidade de Terapia Intensiva de Hospital Universitário*
}

\author{
Laboratory Exams Necessity for Patients Admitted \\ to an University Hospital Intensive Care Unity
}

Fernando Osni Machado, Flávia Solano Patrício da Silva², Juliana Sonego Argente², Rachel Duarte Moritz².

\section{RESUMO}

JUSTIFICATIVA E OBJETIVOS: O aumento progressivo nos recursos diagnósticos tem aumentado a qualidade e a quantidade dos exames de laboratório realizados nas Unidades de Terapia Intensiva (UTI). A influência deste aumento sobre a morbidade e mortalidade não está bem definida. O objetivo deste estudo foi avaliar a freqüência da solicitação de exames na UTI do HU e verificar se houve ou não relação entre a quantidade de exames solicitados e a idade dos pacientes, o seu desfecho e a gravidade das doenças.

MÉTODO: Coorte prospectiva, com abordagem quantitativa. Foram analisados os exames dos pacientes internados na UTI, dos meses de julho a dezembro, 2005. Foram coletados dados clínicos e demográficos dos pacientes e quantificados diariamente os exames mais freqüentemente solicitados na UTI. Seqüencialmente a

1. Doutor em Medicina (USP); Mestre em Medicina Interna (UFSC); Professor do Departamento de Clínica Médica da UFSC

2. Graduando da UFSC.

3. Doutora em Engenharia de Produção - Ergonomia (UFSC); Mestre em Ciências Médicas (UFSC); Professora do Departamento de Clínica Médica da UFSC

*Recebido do Hospital Universitário Professor Polydoro Ernani de São Thiago da Universidade Federal de Santa Catarina (UFSC), Florianópolis, SC.

Apresentado em 13 de outubro de 2006

Aceito para publicação em 11 de dezembro de 2006

Endereço para correspondência:

Dr. Fernando Osni Machado

Av. das Raias, 711/107 - Jurerê Internacional

88053-400 Florianópolis, SC

Fones: (048) 3331-9193, 9980-9389

E-mail: fernandoo.machado@gmail.com

(C)Associação de Medicina Intensiva Brasileira, 2006 média diária de exames foi calculada para todo o período de internação. Para fins de análise os pacientes foram divididos obedecendo três critérios: faixa etária, desfecho de saída da UTI e gravidade. Para a análise estatística foram utilizados os testes Exato de Fisher, Qui-quadrado e ANOVA.

RESULTADOS: Foram admitidos 113 pacientes durante o período de estudo. A taxa média foi de 11,5 exames por dia de internação. Estes valores não apresentaram diferença estatística quando comparados entre os pacientes com idade acima ou abaixo de 60 anos, entre os que sobreviveram e os que foram a óbito e entre aqueles que tiveram taxa de óbito estimada em menos que $50 \%$ ou mais que $50 \%$.

CONCLUSÕES: Os exames solicitados não guardam correlação clínica e prognóstica com sua solicitação. Não houve estatística significativa quando a taxa diária média de exames foi relacionada à idade do paciente, ao desfecho e à gravidade.

Unitermos: custo tratamento, exames complementares, Medicina Intensiva

\section{SUMMARY}

BACKGROUND AND OBJECTIVES: The progressive increasing diagnostic resources had influenced the quality and quantity of laboratory exams. It is not clear if the amount of exams performed influence the morbidity and mortality in the ICU patients. The purpose of this study was to appraise the frequency of the most ordering tests in the ICU of HU-UFSC and to check if there was connection between them and the age, the destiny until the ICU discharge and the estimate severity of their diseases.

METHODS: Prospective cohort study with qualitative approach. The blood samples of admitted patients were analyzed, from July to December 2005. Clinical 
and demographic features were collected and the most frequently blood-samples were quantified per day. In the sequence the daily rate of exams were calculated during all the admission period. The patients were analyzed according to three criterions: age, destiny until the ICU discharge and estimate severity according to APACHE II index. Data were analyzed using Fisher Exact, Chi-square and ANOVA tests.

RESULTS: One hundred and thirteen patients were enrolled to this study. The average test-ordering was 11.50 per day. These numbers didn't have statistical difference when they were compared between survivor and non-survivor patients, and between those whose the death estimated tax was bigger or smaller than 50 per cent.

CONCLUSIONS: The test-ordering didn't show clinical and prognostic relation to its request. There were no statistic relation between the patient's age, ICU discharge and the estimate severity.

Key Words: Intensive Care Medicine, test-ordering, treatment costs.

\section{INTRODUÇÃO}

As unidades de terapia intensiva (UTI) constituem um avanço no cuidado dos pacientes criticamente enfermos. O cuidado intensivo fundamenta-se na tríade: paciente grave, equipamentos de alta tecnologia e equipe multiprofissional especializada ${ }^{1}$.

O perfil dos pacientes que internados nas UTI tem mudado nos últimos anos. O envelhecimento da população tem levado ao aumento da prevalência de doenças crônico degenerativas. As vítimas destas doenças, geralmente com intercorrências agudas, são admitidas cada vez com maior freqüência nas UTI ${ }^{2,3}$.

Os tratamentos nas UTI são onerosos, consumindo entre $25 \%$ e $30 \%$ dos recursos de um hospital ${ }^{4}$. Os gastos com medicação são os que mais consomem recursos financeiros nessas unidades, seguidos da remuneração dos funcionários e da realização de exames complementares ${ }^{5-8}$.

A necessidade da solicitação de exames para pacientes críticos é multifatorial ${ }^{9}$. Pode-se inferir que esse fato seja em conseqüência da maior complexidade das suas doenças. Entretanto, a facilitação da realização dos exames e os fatores culturais do médico intensivista possivelmente estão também relacionados ao número de exames solicitados ${ }^{4,10-12}$.

O objetivo deste estudo foi avaliar a freqüência da solicitação de exames para os pacientes internados na
UTI. Secundariamente, foi verificado se houve ou não relação entre a idade dos pacientes, a gravidade das suas doenças e a quantidade de exames solicitados.

\section{MÉTODO}

Após aprovação do Comitê de Ética Médica em Pesquisa em seres humanos do Hospital Universitário Polydoro Ernani de São Thiago (HU) da Universidade Federal de Santa Catarina (UFSC), foi realizado um estudo de corte prospectivo, não controlado, com abordagem quantitativa, sob protocolo de $n^{\circ} 192 / 05$ de 27 de junho de 2005.

A população do estudo foi constituída pelos pacientes que foram internados na UTI/HU no período de 6 de julho a 6 de dezembro de 2005. Foram excluídos os pacientes transferidos, enquanto internados na UTI, para outros hospitais.

Os dados coletados foram obtidos através da consulta ao prontuário do paciente a partir do momento da sua internação na UTI, tais como número do prontuário, sexo, idade, data da admissão e da alta na UTI, desfecho de saída dos pacientes da UTI (alta ou óbito), o índice e o escore APACHE II e o número de exames solicitados durante a internação.

Os exames quantificados foram aqueles que mais comumente são solicitados em UTI: sódio, potássio, cálcio, fósforo, magnésio, creatinina e uréia séricos, tempo de protrombina (TAP), tempo de tromboplastina parcial ativada (TTPA), ácido lático, gasometria arterial, glicemia capilar, glicemia de jejum, hemograma e plaquetas.

Para análise estatística os pacientes foram divididos obedecendo três critérios: faixa etária (menor ou igual e maior que 60 anos), desfecho de saída da UTI (alta ou óbito), e gravidade (índice APACHE II, tendo como ponto de corte $50 \%)^{13}$.

As variáveis contínuas foram analisadas com o uso do teste de Análise de Variância (ANOVA) e para avaliar proporções utilizou-se, quando necessário, os testes Qui-quadrado e Exato de Fisher, sendo considerado significativo quando $p<0,05$.

\section{RESULTADOS}

Durante o período do estudo, foram internados, na UTI do HU/UFSC, 116 pacientes com faixa etária entre 15 e 89 anos. Desses pacientes, três foram transferidos para a UTI de outros hospitais. Portanto, 113 pacientes perfizeram a amostra deste estudo. 
Foram solicitados para esses pacientes uma média de 11,5 exames/dia. Os mais freqüentemente solicitados foram glicemia capilar $(25,5)$, gasometria arterial $(7,6) \mathrm{e}$ potássio sérico $(6,5)$ exames/dia.

A tabela 1 apresenta as variáveis dos pacientes relacionadas à idade. Na tabela 2, essas variáveis aparecem relacionadas ao desfecho dos pacientes na UTI (alta ou óbito). Na tabela 3 são destacadas as variáveis relacionadas à gravidade do quadro clínico, verificada através do índice APACHE II, sendo considerado como ponto de corte a previsão de mortalidade de $50 \%$.
Pode-se constatar que não houve estatística significativa em relação à quantidade de exames solicitados no que concerne a idade, desfecho dos pacientes (alta/ óbito) e índice APACHE II.

\section{DISCUSSÃO}

A política e o aperfeiçoamento tecnológico na área da saúde tem levado ao aumento da longevidade da população, com conseqüente maior gasto financeiro. A maioria dos trabalhos mundiais que abordam esse tema avalia o custo-efetividade dos tratamen-

Tabela 1 - Comparação das Variáveis Avaliadas de acordo com a Idade dos Pacientes $(n=113)$.

\begin{tabular}{|c|c|c|c|c|}
\hline Variáveis & Total de Pacientes & Pacientes $<60$ Anos & Pacientes $\geq 60$ Anos & $p$ \\
\hline $\mathrm{N}^{\circ}$ de pacientes & 113 & 61 & 52 & - \\
\hline \multicolumn{5}{|l|}{ Sexo } \\
\hline Masculino & 68 & 36 & 32 & NS \\
\hline \multicolumn{5}{|l|}{ Desfecho } \\
\hline Alta & 79 & 43 & 36 & NS \\
\hline Índice APACHE II (\% média) & 44,9 & 41,8 & 48,4 & NS \\
\hline Tempo médio de internação UTI (dias) & 7,2 & 6,7 & 7,8 & NS \\
\hline Taxa do $n^{\circ}$ de exames/dia (média) & 11,5 & 11,4 & 11,8 & NS \\
\hline
\end{tabular}

NS = não significativos

Tabela 2 - Comparação das Variáveis Analisadas de acordo com o Desfecho dos Pacientes.

\begin{tabular}{|c|c|c|c|c|}
\hline Variáveis & Total de Pacientes & Alta & Óbito & $p$ \\
\hline Número de pacientes & 113 & 79 & 34 & - \\
\hline Idade (anos) & 54,4 & 54,1 & 55,0 & NS \\
\hline \multicolumn{5}{|l|}{ Sexo } \\
\hline Feminino & 45 & 31 & 14 & NS \\
\hline Índice APACHE II (médio) & 22 & 18,7 & 28,9 & $<0,05$ \\
\hline Taxa do $n^{\circ}$ de exames/dia (média) & 11,6 & 11,3 & 12 & NS \\
\hline
\end{tabular}

NS = não significativos

Tabela 3 - Variáveis Relacionadas à Gravidade dos Pacientes (Índice APACHE II).

\begin{tabular}{|c|c|c|c|c|}
\hline Variáveis & Total de Pacientes & Índice APACHE II <50\% & Índice APACHE II $\geq 50 \%$ & $\mathrm{p}$ \\
\hline Número de pacientes & 113 & 78 & 35 & - \\
\hline Idade (anos) & 54,4 & 52,4 & 59,7 & NS \\
\hline \multicolumn{5}{|l|}{ Sexo } \\
\hline Masculino & 68 & 46 & 22 & NS \\
\hline Feminino & 45 & 32 & 13 & NS \\
\hline \multicolumn{5}{|l|}{ Desfecho } \\
\hline Alta & 79 & 65 & 14 & $<0,05$ \\
\hline Óbito & 34 & 13 & 21 & \\
\hline Tempo médio de internação UTI (dias) & 7,2 & 6,7 & 8 & NS \\
\hline Taxa do $n^{\circ}$ de exames/dia (média) & 11,6 & 11,03 & 12,9 & NS \\
\hline
\end{tabular}

NS = não significativos 
tos em UTI, assim como a adesão de protocolos que promovam a sua redução ${ }^{10,13-17}$. Entretanto, são poucos os estudos que abordam especificamente a necessidade da realização de exames solicitados nas UTI 12,18 .

Neste estudo constatou-se que foram solicitados, em média, 11,5 exames/dia. Resultados semelhantes foram descritos na literatura revisada. Zimmerman e col. ${ }^{19}$ analisaram a freqüência de solicitação de exames laboratoriais em 42 UTI norte-americanas, e evidenciaram que nos hospitais de ensino são realizados, em média, no primeiro e no segundo dia de internação 12,8 e 7,6 exames. Os autores apontaram que esse número foi duas vezes maior que o encontrado na análise de hospitais que não se destinavam à formação acadêmica. Nguyen e col. ${ }^{20}$ constataram média diária de 11,7 exames realizados em hospital de excelência para a realização de pesquisas em cuidados intensivos na Bélgica. Ressalta-se que este estudo foi realizado em hospital universitário. O excessivo número de exames laboratoriais solicitados nos hospitais de ensino pode ser atribuído a inúmeros fatores. Dentre os mais importantes pode-se destacar a inexperiência dos médicos em treinamento e os questionamentos freqüentes desses profissionais a seus professores, havendo em conseqüência desse fato maior necessidade de fundamentação teórico e prática diante de decisões a serem tomadas.

Neste estudo, levando-se em consideração a gravidade das doenças dos pacientes e a quantidade de exames solicitados, pode-se constatar que não houve relação significativa entre esses fatos. Da mesma forma, foi possível constatar que a idade não representou fator determinante para a solicitação de exames. Resultados diferentes foram apontados por Zimmerman e col. ${ }^{19}$ que avaliaram 17.440 pacientes e constataram que o número de exames de sangue foi maior entre os pacientes com condições clínicas mais graves, verificadas através do índice APACHE III.

As causas de admissão e a gravidade das doenças dos pacientes internados em UTI são diversas. Portanto, seria lógico que houvesse relação direta entre esses fatos, a quantidade e a qualidade de exames solicitados, fato que não ocorreu neste estudo. Poderia se inferir que existem exames considerados rotineiros e que os médicos sintam-se compelidos a solicitá-los, principalmente quando prestam atendimento a pacientes criticamente enfermos. Entretanto, deve-se enfatizar que neste trabalho não foi observa- da nenhuma influência no que concerne a quantidade de exames solicitados e ao desfecho dos pacientes. A partir desses dados surge o questionamento sobre a real importância de uma rotina de solicitação de exames laboratoriais. Pode-se também questionar se o hábito freqüente da solicitação conjunta de certos exames, como sódio e o potássio, é inadequado, pois a alteração em um deles não leva necessariamente à alteração do outro.

Em estudo realizado na Austrália e na Nova Zelândia, Flabouris e col. ${ }^{10}$ compararam a solicitação de exames e concluíram que, mesmo nas UTI que seguiam protocolos, os exames mais solicitados foram a gasometria arterial, o hemograma e a dosagem de eletrólitos. Os mesmos resultados foram apontados por Barie e Hydo ${ }^{17}$, em UTI cirúrgica dos Estados Unidos. Os resultados deste trabalho corroboram com os já mencionados, com exceção da glicemia capilar, que se apresentou como o exame mais solicitado na UTI/ HU. Esse exame não foi mencionado nos trabalhos revisados. Pode-se explicar esse fato, por ser a glicemia capilar um teste realizado à beira do leito fato que pode ter contribuído para que ele não tenha sido apontado em outros estudos. Deve-se ressaltar que a avaliação da glicemia capilar é freqüentemente indicada para o manuseio do paciente gravemente enfermo e necessita ser realizada em intervalo que varia de uma hora ou até cada seis horas.

É natural que a aplicação de recursos financeiros seja diferenciada para os pacientes, de acordo com o quadro clínico e gravidade das suas doenças ${ }^{4}$. A solicitação de exames é diretamente proporcional ao custo dispensado à sua realização, como foi evidenciado em estudos que analisaram o custo-efetividade dos cuidados em UTI 4,15. O impacto financeiro provocado pela redução do número de exames solicitados pode levar a uma economia de cerca de $\$ 14,22$ dólares (em 1998), por dia, para cada paciente ${ }^{15}$. A percepção dos médicos de que o número excessivo de solicitações de exames de sangue é diretamente proporcional ao custo financeiro do tratamento, permite a sugestão de que essa prática deva ser modificada ${ }^{21}$. A inclusão de diretrizes para a solicitação de exames em UTI da Nova Zelândia revelou um decréscimo de $16,6 \%$ no total de exames e de $21,9 \%$ na solicitação de gasometrias arteriais ${ }^{13}$. Blackstone e col. ${ }^{16}$ concluíram que e a utilização de um protocolo de solicitação de exames, baseado nas condições clínicas do paciente, resultou num decréscimo nos gastos diários, sem haver influência na morbimorta- 
lidade dos pacientes. Barie e Hydo ${ }^{17}$ estabeleceram significativa diminuição dos custos de UTI cirúrgica com a conscientização dos médicos que nela trabaIhavam, sobre aos gastos relativos às realizações de exames complementares.

Pode-se constatar neste estudo que os exames foram solicitados independentemente de índices prognósticos e sem interferência com a evolução dos pacientes. Tal prática se refletiu em gastos que poderiam ser reduzidos se a solicitação de exames fosse definida através de diretrizes baseadas em critérios clínicos e prognósticos, visando a avaliação criteriosa e individualizada quanto as necessidades reais de cada paciente.

Tal como observado em outros trabalhos científicos, pode-se atribuir algumas limitações deste estudo. A primeira delas é quanto ao tamanho limitado da amostra, que pode ser atribuído ao número reduzido de leitos disponíveis na UTI do HU/UFSC e à média de dias em que estes leitos estiveram ocupados por um único paciente. Em segundo lugar encontra-se a dificuldade em avaliar o custo real da realização dos exames a que se destina o estudo, já que não foram computados os valores referentes aos tubos de coleta, seringas e demais materiais necessários para a sua execução e para a análise do sangue coletado. Quanto às implicações deste trabalho para a prática clínica e pesquisa científica, o conhecimento de que o número excessivo de exames pode levar a custos desnecessários e a possíveis repercussões clínicas, poderá justificar a possível reflexão a respeito da adoção de parâmetros que objetivem a restrição de solicitação destes exames ${ }^{13,15}$.

\section{AGRADECIMENTOS:}

Agradeço ao Dr. Antonio Carlos Estima Marasciolo, pelo relevante auxílio na análise estatística.

\section{REFERÊNCIAS}

01. Couto RC, Botoni FA, Serufo JC et al - Ratton-Emergências Médicas e Terapia Intensiva. Rio de Janeiro: Guanabara Koogan, 2005.

02. Kleinpell RM, Ferrans CE - Factor influencing intensive care unit survival for critically ill elderly patients. Heart Lung, 1998;27:337-343.

03. Fedullo AJ, Swinburn AJ - Relationship of patient age to cost and survival in a medical ICU. Crit Care Med, 1983;11:155-159.

04. Chalfin DB, Cohen IL, Lambrinos J - The economics and cost-effectiveness of critical care medicine. Intensive Care Med, 1995;11:952-961.

05. Krinsley JS - Test-ordering strategy in the intensive care unit. Intensive Care Med, 2003;18:330-339.

06. Moerer O, Shimd A, Hofmann M et al - Direct costs of severe sepsis in the three German intensive care units based on retrospective electronic patient record analysis of resources use. Intensive Care Med, 2002;28:1440-1446.

07. Wisser D, Ackern K, Knoll E et al - Blood loss from laboratory tests. Clinical Chemistry, 2003;49:1651-1655.

08. Beland D, D'Angelo C, Vinci D - Reducing unnecessary blood working in the neurosurgical ICU. Neurosci Nurs, 2003;35:149-152.

09. Goldman L, Bennett JC - Cecil Tratado de Medicina Interna. $21^{\text {a }}$ Ed, Rio de Janeiro: Guanabara Koogan, 2001.

10. Flabouris A, Bishop G, Williams $L$ et al - Routine blood test ordering for patients in the intensive care. Anaesth Intens Care, 2000;28:562-565.

11. Barba EJR - Utilización inapropiada del laboratorio clínico. Rev Mex Patol Clin, 2003;50:209-223.

12. Santos AACS, Godoy MF - Análise crítica da solicitação de exames complementares em pediatria. Pediatria, 1999;2:215-221.

13. Mehari SM, Havill JH, Montgomery C - A written guideline implementation can lad to reductions in the laboratory testing in an intensive care unit. Anaesth Intens Care, 1997;25:33-37.

14. Beland D, D'Angelo C, Vinci D - Reducing unnecessary blood working in the neurosurgical ICU. Neurosci Nurs, 2003;35:149-152.

15. Wang TJ, Mort EA, Nordberg P - A utilization management intervention to reduce unnecessary testing in the coronary care unit. Arch Intern Med, 2002;162:1885-1890.

16. Blackstone ME, Miller S, Hodgson AJ et al - Lowering hospital charges in the Trauma Intensive Care Unit while maintaining quality of care by increasing resident and attending physician awareness. J Trauma, 1995;39:1041-1044.

17. Barie PS, Hydo LJ - Lessons learned: durability and progress of a program for ancillary cost reduction in surgical critical care. J Trauma, 1997;43:590-594.

18. Lingtenberg JJM, Meijering S, Stienstra $Y$ et al - Mean glucose level is not an independent risk factor for mortality in mixed ICU patients. Intensive Care Med, 2006;32:435-438.

19. Zimmerman JE, Seneff MG, Sun $X$ et al - Evaluating laboratory usage in the intensive care unit: patient and institutional characteristics that influence frequency of blood sampling. Crit Care Med, 1997;25:737-748.

20. Nguyen V, Bota DP, Melot C et al - Time course of hemoglobin concentrations in nonbleeding intensive care unit patients. Crit Care Med, 2003;31:406-410.

21. Cook DJ - Physicians' perceptions of laboratory costs in the intensive care unit: Hamilton Regional Critical Care Group. Clin Invest Med, 1992;15:476-481. 